\title{
PLANTATION OF A NEW FORMULA TO ASSESS PURITY OF FINAL MOLASSES
}

\author{
Hayat-ur-Rahim Khan* and Areeba Tehreem** \\ *Technical Director, Ashraf Sugar Mills Ltd. Bahawalpur \\ ${ }^{* *}$ Comsats University, Lahore \\ Email: hayat.salaarzai@gmail.com
}

\section{ABSTRACT}

Loss of sucrose in final molasses is a major financial loss to a sugar factory; all efforts are to be made to curtail this loss. The apparent purity concept is unable to quantify the degree of molasses exhaustion but the Target Purity Formula can be used as bench mark to fulfill this purpose. Target Purity formula is a function of mono saccharides and inorganic components. A number of Target Purity Formulas have been presented in the past. Thirty tests of analysis of molasses exhaustion were collected from various sugar mills of the country in three years. All available Target Purity Formula applied on these tests to find an appropriate formula which can be used to quantify the exhaustion of final molasses for our country. Evaluating some of the Target Purity Formulas, a new formula is being planted (40.86-14.60 log Rs/A) and checked against all thirty tests analysis of final molasses exhaustion and found suitable for our country.

\section{INTRODUCTION}

The loss of sucrose in final molasses is the biggest loss which has to suffer by a Sugar Factory. This loss has a significant impact on the profitability of a Sugar Mills directly or indirectly. It is essential to obtain a reliable data on final molasses exhaustion to minimize sucrose loss. The normal measurement or apparent purity obtained in Sugar Mills Laboratory is not accurate enough to check sugar loss in final molasses. To assess the degree of exhaustion achievable, a "Bench Mark" is required. Which is luckily available in the form of "Target Purity" formula.
Target purity is the lowest final molasses purity achievable in a Sugar factory. It reflects the dependence of optimum final molasses exhaustion on the ratio of two main factors Reducing Sugar (RS) and Ash (A) ratio. Presence of reducing sugar (mono saccharides) or Fructose + Glucose in the juice has an ability to decrease the solubility of sucrose in the molasses while inorganic components (Ash) tend to increase solubility. RS/A ratio are used as the criterion for molasses exhaustion. It is the only factor that effects the purity of final molasses during the processing of juices and mother liquor.

The aim of this study is to find out appropriate target purity equation that can be used to quantify molasses exhaustion in our country because apparent purity concept is considered insufficient to quantify the degree of molasses exhaustion. Over the years, a number of different Target Purity Formulas have been presented for different cane growing areas of the world. So, it has become a necessity to choose a most appropriate equation for our country also. 
Target purity equations used in different parts of the world are given as under:

\begin{tabular}{|c|c|}
\hline Foster (1960) & $40.7-17.8 . \log \left(q_{R S / A}\right)$ \\
\hline Miller et al. (1998) (No. 1) & $41.1-8.7 . \log \left(q_{R S} / A\right)$ \\
\hline Miller et al. (1998) (No. 2) & $46.9-9.5 .\left[1-\exp \left(-1.3 . q_{M S} / \mathrm{A}\right)\right]$ \\
\hline Miller et al. (1998) (No. 3) & $39.4-10.6 . \log \left(q_{R S / A}\right)$ \\
\hline Miller et al. (1998) (No. 4) & 55.1-18.7. [1-exp $(-2.6 . q M S / A)]$ \\
\hline Bruijn et al. (1972) & $39.9-19.6 . \log \left(q_{R S / A}\right)$ \\
\hline Rein and Smith (1981) (No.1) & $37.7-17.6 . \log \left(q_{R S / A}\right)$ \\
\hline Rein and Smith (1981) (No.2) & $33.9-13.4 . \log \left(\mathrm{q}_{\mathrm{MS} / \mathrm{A}}\right)$ \\
\hline Smith (1995) & $43.1-17.5 .[1-\exp (-0.74 . \mathrm{qmS} / \mathrm{A})]$ \\
\hline Saska et al. (1999) & $42.4-12.3 . \log (\mathrm{qms} / \mathrm{A})$ \\
\hline Gil et al. (2001) (No. 1) & $38.5-7.7 . \ln (\mathrm{qRS} / \mathrm{A})$ \\
\hline Gil et al. (2001) (No. 2) & $35.8-6.3 . \ln \left(q_{R S / A}\right)$ \\
\hline
\end{tabular}

Where

$\mathrm{RS} / \mathrm{A}=$ Reducing Sugar Ash Ratio

$\mathrm{MS} / \mathrm{A}=$ Mono Saccharide Ash Ratio

\section{Review of Literature}

The principal loss of sucrose in the boiling house is that retained in the final molasses. As sucrose crystallization from final molasses proceeds, the rate of crystallization becomes slower and slower until finally a point is reached at which no more sucrose will crystallize out at a given temperature. The molasses then is termed exhausted. The purity of exhausted molasses depends principally on water content. At the same water content, however, the nature and quantity of non-sucrose constituents, having their origin in cane, govern the purity; of these reducing sugars and ash have major controlling effect (Payne, 1982).

Hugot (1986) gives a simple formula of expected purity of final molasses used in the
Reunion i.e. expected purity $=40-4 \times$ Reducing sugars.

Many operating factors, such as the character of the crystals and density of the final massecuites, the extent of the cooling in the crystallizers, and the after treatment of massecuite, effect the purity of final molasses. Viscosity is also of great importance in exhaustion of molasses, since it is one of the factors that limit the concentration of the massecuite and the super saturations of the molasses (Meade,1964).

Most of the sugar factories estimate their performance by the apparent purity of final molasses. However, this method of comparison in the same factory is doubtful value. With the progress of the crop and maturing of the cane the relation of sucrose to reducing sugars changes and therefore direct polarization will change and consequently apparent purity. Since in a given sugar factory the ash content in molasses varies very little, it can be disregarded for all practical purposes, and only the sucrose reducing sugars and Brix of molasses can be taken into consideration for calculation of maximum possible exhaustion of molasses (Baikow, 1967).

\section{Target Purity Formula} Calculation

Material and Method:

In order to calculate Target Purity formula following steps were adopted in SSML laboratory.

Apparent Brix of Molasses:

Apparent Pol of Molasses:

Apparent Purity of Molasses:

Determination of Sulphated Ash in Final Molasses: 
Heat a platinum dish to redness in the furnace.

Transfer to a desiccator and allow cooling. Weigh the dish on analytical balance.

Transfer 3-5 $\mathrm{g}$ of molasses to the dish and weigh. Add $5 \mathrm{ml}$ of 10 percent sulphuric acid.

Evaporate on a steam bath for 2-3 hours and then heat on hot plate until completely charred. Start hot plate on low heat and gradually increase to high heat to prevent spattering of sample.

Transfer to the furnace and heat at $550^{\circ} \mathrm{C}$ for five hours.

Remove from furnace; cool and carefully add $3 \mathrm{ml}$ of 10 percent sulphuric acid wetting the entire dish. Evaporate almost to dryness on steam bath and then heat on hot plate until sulphuric acid is volatilized. Transfer to furnace again and heat at $500^{\circ} \mathrm{C}$ for two hours.

Remove from furnace, cool in a desiccator and weigh.

Calculation and Example:

Table:

\begin{tabular}{|l|l|}
\hline Burette Reading & Value \\
\hline 20.0 & 50.9 \\
\hline 21.0 & 51.00 \\
\hline 22.0 & 51.10 \\
\hline 23.0 & 51.10 \\
\hline 24.0 & 51.20 \\
\hline 25.0 & 51.20 \\
\hline 26.0 & 51.30 \\
\hline 27.0 & 51.40 \\
\hline
\end{tabular}

B. Reading $=26$ $\begin{aligned} \text { Total Sugar } & =\frac{51.30 \times 200}{52.62} \\ & =52\end{aligned}$
Calculation and Example:

Weight of empty platinum dish $=$

Weight of dish plus molasses $=37.332 \mathrm{~g}$

Weight of molasses sample Weight of dish plus ash after ignition $=33.533 \mathrm{~g}$

Weight of ash 33.53332.582

Sulphated Ash \% = 20.02

True Solids $=$ Brix

Molasses - $(0.74 \quad x$

Sulphated Ash)

True Purity =

Reducing Sugar (Eynon \& Lane Method)

Take $5 \mathrm{ml}$ A-Fehling Solution $+5 \mathrm{ml} \mathrm{B}$-Fehling Solution in a Conical Flask.

Add $30 \mathrm{ml}$ Distilled Water

Add 2-3 drops of methylenblau as indicator and mix well.

Heat on hot plate and titrate against Final Molasses and note the burette reading.
Calculation R.S. \% =

Total Sugar as invert in Final Mogassios g

Wt. $0.75 \mathrm{gm}$ of molasses sample in a $100 \mathrm{ml}$ beaker on analyticałbaletedeg.

Add $20-30 \mathrm{ml}$ of Distilled Water and mix well.

Transfer into a $200 \mathrm{ml}$ flask and fill $1 / 2=$ not $155 / i s g i l l e d$ water.

Cool on room temperature for $1 / 2$ hours.

Add 4-5 drops of P.P. as indicator.

Add 10 Sererd\$AQH0Q until pink color appears.

Complete the volume with distilled water.

Take $5 \mathrm{ml} \mathrm{A-Fehling} \mathrm{solution}$ + $5 \mathrm{ml}$ B-Fehling solution into a conical flask add $15-20 \mathrm{ml}$ distilled water and 2-3 drops of methylenblau.

Titrate against final molasses and note the burette reading. 
various sugar mills all over the country in three years during season 2012-13, 2013-14 \& 2014-

2015 calculated according to twelve Target Purity Equations.

Results are shown in Table No. 1, 2, \& 3 .

\section{Choice of Target Purity Formula:}

Thirty exhaustion molasses tests were collected from various Sugar Mills all over the country in three years during season 2012-13, 2013-14 \& 2014-2015 calculated according to twelve Target Purity Equations. Results are shown in Table No. 1,2, \& 3.

\section{Plantation of a New Formula:}

It is obvious from Table No. $1,2 \& 3$ that results of target purity and true purity difference (TPD) of following four equations are very marginal as compared to other eight equations.

Foster 40.7-17.8 log RS/A Near or Equal 16 Results

Miller 41.1-8.7 log RS/A Near or Equal 14 Results

Bruijin 39.9-19.6 log RS/A Near or Equal 20 Results

Saska 42.4-12.3 log RS/A Near or Equal 10

Results

If all these four equations are evaluated in one equation, a new formula is formed: 40.86-14.60 Log RS/AB. Reading $=26$

Total Sugar $=$ Thirty exhaustion molasses tests were collected from various sugar mills all over the country in three years during season 2012-13, 2013-14 \& 2014-2015 calculated according to twelve Target Purity Equations. $\frac{51.30 \times 200}{0.26 \times .75}$ $=52.6210 \times 26 \times .75$

Sucrose $\%=$ Total Sugar as invert - R.S. X 0.95

\section{Choice of Target Purity} Formula:

Thirty exhaustion molasses tests were collected from various sugar mills all over the country in three years during season 2012-13, 2013-14 \& 2014-2015 calculated according to twelve Target Purity Equations.

Results are shown in Table No. 1, 2, \& 3 .

\section{Choice of Target Purity Formula:}

Thirty exhaustion molasses tests were collected from various Sugar Mills all over the country in three years during season 2012-13, 2013-14 \& 2014-2015 calculated according to twelve Target Purity Equations. Results are shown in Table No. 1,2, \& 3.

\section{Plantation of a New Formula:}

It is obvious from Table No. 1,2 \&3 that results of target purity and true purity difference (TPD) of following four equations are very marginal as compared to other eight equations.

Foster 40.7-17.8 log RS/A Near or Equal 16 Results

Miller 41.1-8.7 log RS/A Near or Equal 14 Results

Bruijin 39.9-19.6 log RS/A Near or Equal 20 Results

Saska

If all these four equations are evaluated in one equation, a new formula is formed: $40.86-14.60$ Log RS/A 
Table No. 1 Season 2012-13

\begin{tabular}{|c|c|c|c|c|c|c|c|c|c|c|c|c|c|c|c|c|c|c|}
\hline \multirow[t]{2}{*}{$\begin{array}{c}\text { Factory } \\
\# .\end{array}$} & \multirow[t]{2}{*}{$\begin{array}{l}\text { Reducing } \\
\text { Sugar }\end{array}$} & \multirow[t]{2}{*}{$\begin{array}{c}\text { Sulphated } \\
\text { Ash }\end{array}$} & \multirow[t]{2}{*}{$\begin{array}{l}\text { RS/A } \\
\text { Ratio }\end{array}$} & \multirow[t]{2}{*}{$\begin{array}{l}\text { True } \\
\text { Solids }\end{array}$} & \multirow[t]{2}{*}{ Sucrose } & \multirow[t]{2}{*}{$\begin{array}{l}\text { True } \\
\text { Pty }\end{array}$} & \multirow{2}{*}{ 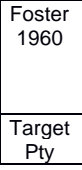 } & \multirow{2}{*}{$\begin{array}{c}1 . \\
\text { Miller } \\
1998 \\
\\
\begin{array}{c}\text { Target } \\
\text { Pty }\end{array} \\
\end{array}$} & \multirow{2}{*}{\begin{tabular}{|c|}
$\begin{array}{c}\text { 2. Miller } \\
1998\end{array}$ \\
$\begin{array}{c}\text { Target } \\
\text { Pty }\end{array}$ \\
\end{tabular}} & \multirow{2}{*}{\begin{tabular}{|c|} 
3. Miller \\
1998 \\
\\
$\begin{array}{c}\text { Target } \\
\text { Pty }\end{array}$ \\
\end{tabular}} & \multirow{2}{*}{ 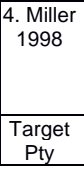 } & \multirow{2}{*}{\begin{tabular}{|c|} 
Brujin \\
1972 \\
\\
$\begin{array}{c}\text { Target } \\
\text { Pty }\end{array}$ \\
\end{tabular}} & \multirow{2}{*}{\begin{tabular}{|l|}
$\begin{array}{l}1 . \text { Rein } \\
\& \text { Smith } \\
1981\end{array}$ \\
$\begin{array}{c}\text { Target } \\
\text { Pty }\end{array}$ \\
\end{tabular}} & \multirow{2}{*}{\begin{tabular}{|l|}
$\begin{array}{l}\text { 2.Rein } \\
\& \text { Smith } \\
1981\end{array}$ \\
$\begin{array}{c}\text { Target } \\
\text { Pty }\end{array}$ \\
\end{tabular}} & \multirow{2}{*}{$\begin{array}{c}\text { Smith } \\
1995 \\
\\
\text { Target } \\
\text { Pty } \\
\end{array}$} & \multirow{2}{*}{ 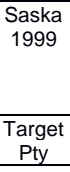 } & \multirow{2}{*}{\begin{tabular}{|l|}
$\begin{array}{l}1 . G i l \text { et } \\
\text { al } 2001\end{array}$ \\
$\begin{array}{c}\text { Target } \\
\text { Pty }\end{array}$ \\
\end{tabular}} & \multirow{2}{*}{\begin{tabular}{|l|}
$\begin{array}{l}\text { 2.Gil et } \\
\text { al } 2001\end{array}$ \\
$\begin{array}{c}\text { Target } \\
\text { Pty }\end{array}$ \\
\end{tabular}} \\
\hline & & & & & & & & & & & & & & & & & & \\
\hline 1 & 14.76 & 14.96 & 0.99 & 85.43 & 31.43 & 36.79 & 40.80 & 40.80 & 41.15 & 39.46 & 37.84 & \begin{tabular}{|l|}
40.01 \\
\end{tabular} & 37.80 & 33.98 & 34.03 & \begin{tabular}{|l|}
42.47 \\
\end{tabular} & 38.60 & 35.88 \\
\hline 2 & 13.77 & 15.00 & 0.92 & 88.24 & 31.75 & 35.98 & 41.36 & 41.36 & 41.42 & 39.79 & 38.12 & \begin{tabular}{|l|}
40.63 \\
\end{tabular} & 38.35 & 34.40 & 34.47 & 42.86 & 39.16 & 36.34 \\
\hline 3 & 11.90 & 18.23 & 0.65 & 77.30 & 34.29 & 44.36 & 44.00 & 44.00 & 42.71 & 41.36 & 39.83 & \begin{tabular}{|l|}
43.53 \\
\end{tabular} & 40.96 & 36.38 & 36.40 & \begin{tabular}{|l|}
44.68 \\
\end{tabular} & 41.78 & 38.49 \\
\hline 4 & 14.40 & 18.27 & 0.79 & 85.54 & 36.67 & 42.87 & 42.54 & 42.54 & 42.00 & 40.50 & 38.81 & \begin{tabular}{|l|}
41.93 \\
\end{tabular} & 39.52 & 35.29 & 35.37 & \begin{tabular}{|l|}
43.67 \\
\end{tabular} & 40.33 & 37.30 \\
\hline 5 & 13.78 & 16.79 & $\begin{array}{l}0.82 \\
\end{array}$ & 78.17 & 32.03 & 40.97 & 42.23 & 42.23 & 41.85 & 40.31 & 38.61 & \begin{tabular}{|l|}
41.58 \\
\end{tabular} & 39.21 & 35.05 & 35.13 & \begin{tabular}{|l|}
43.46 \\
\end{tabular} & 40.02 & 37.04 \\
\hline 6 & 12.01 & 18.40 & 0.65 & 81.28 & 35.14 & 43.23 & 44.00 & 44.00 & 42.71 & 41.36 & 39.83 & \begin{tabular}{|l|}
43.53 \\
\end{tabular} & 40.96 & 36.38 & 36.40 & \begin{tabular}{|l|}
44.68 \\
\end{tabular} & 41.78 & 38.49 \\
\hline 7 & 14.12 & 16.90 & $\begin{array}{l}0.84 \\
\end{array}$ & 80.09 & 36.40 & 45.45 & 42.09 & 42.09 & 41.78 & 40.23 & 38.53 & \begin{tabular}{|l|l}
41.43 \\
\end{tabular} & 39.07 & 34.95 & 35.03 & \begin{tabular}{|l|}
43.36 \\
\end{tabular} & 39.88 & 36.93 \\
\hline 8 & 13.03 & 17.18 & 0.76 & 85.18 & 36.40 & 42.73 & 42.84 & 42.84 & 42.14 & 40.67 & 39.00 & \begin{tabular}{|l|}
42.25 \\
\end{tabular} & 39.81 & 35.51 & 35.58 & \begin{tabular}{|l|}
43.88 \\
\end{tabular} & 40.63 & 37.54 \\
\hline 9 & 12.09 & 17.45 & 0.69 & 87.48 & 37.77 & 43.18 & 43.54 & 43.54 & 42.49 & 41.09 & 39.49 & \begin{tabular}{|l|}
43.02 \\
\end{tabular} & 40.50 & 36.04 & 36.08 & \begin{tabular}{|l|}
44.36 \\
\end{tabular} & 41.33 & 38.11 \\
\hline 10 & 12.20 & 16.33 & 0.75 & 86.41 & 37.96 & 43.93 & 42.95 & 42.95 & 42.20 & 40.74 & 39.08 & \begin{tabular}{|l|}
42.38 \\
\end{tabular} & 39.93 & 35.60 & 35.67 & \begin{tabular}{|l|}
43.96 \\
\end{tabular} & 40.75 & 37.64 \\
\hline 11 & 12.87 & 17.66 & 0.73 & 86.03 & 37.31 & 43.37 & 43.15 & 43.15 & 42.30 & 40.86 & 39.21 & 42.59 & 40.12 & 35.74 & 35.81 & 44.09 & 40.94 & 37.79 \\
\hline 12 & 11.96 & 18.07 & 0.66 & 87.45 & 37.70 & 43.11 & 43.89 & 43.89 & 42.66 & 41.30 & 39.75 & \begin{tabular}{|l|}
43.41 \\
\end{tabular} & 40.85 & 36.30 & 36.32 & \begin{tabular}{|l|l|}
44.60 \\
\end{tabular} & 41.68 & 38.40 \\
\hline 13 & 15.13 & 15.60 & 0.97 & 86.08 & 37.57 & 43.65 & 40.94 & 40.94 & 41.22 & 39.54 & 37.90 & \begin{tabular}{|l|}
40.16 \\
\end{tabular} & 37.93 & 34.08 & 34.14 & \begin{tabular}{|l|}
42.56 \\
\end{tabular} & 38.74 & 35.99 \\
\hline 14 & 14.67 & 16.60 & 0.88 & 84.79 & 33.51 & 39.52 & 41.66 & 41.66 & 41.57 & 39.97 & 38.28 & 40.95 & 38.64 & 34.62 & 34.70 & 43.06 & 39.45 & 36.58 \\
\hline 15 & 15.10 & 14.60 & 1.03 & 86.49 & 32.17 & 37.20 & 40.44 & 40.44 & 40.97 & 39.24 & 37.67 & 39.61 & 37.44 & 33.70 & 33.74 & 42.22 & 38.24 & 35.59 \\
\hline
\end{tabular}

Table No. 2

Season 2013-14

\begin{tabular}{|c|c|c|c|c|c|c|c|c|c|c|c|c|c|c|c|c|c|c|}
\hline \multirow[t]{2}{*}{$\begin{array}{c}\text { Factory } \\
\# .\end{array}$} & \multirow[t]{2}{*}{$\begin{array}{c}\text { Reducing } \\
\text { Sugar }\end{array}$} & \multirow[t]{2}{*}{$\begin{array}{c}\text { Sulphated } \\
\text { Ash }\end{array}$} & \multirow[t]{2}{*}{$\begin{array}{l}\mathrm{RS} / \mathrm{A} \\
\text { Ratio }\end{array}$} & \multirow[t]{2}{*}{$\begin{array}{l}\text { True } \\
\text { Solids }\end{array}$} & \multirow[t]{2}{*}{ Sucrose } & \multirow[t]{2}{*}{$\begin{array}{l}\text { True } \\
\text { Pty }\end{array}$} & \multirow{2}{*}{ 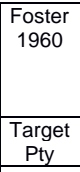 } & \multirow{2}{*}{$\begin{array}{c}1 . \\
\text { Miller } \\
1998 \\
\\
\begin{array}{c}\text { Target } \\
\text { Pty }\end{array} \\
\end{array}$} & \multirow{2}{*}{\begin{tabular}{|c|} 
2. Miller \\
1998 \\
\\
\\
$\begin{array}{c}\text { Target } \\
\text { Pty }\end{array}$ \\
\end{tabular}} & \multirow{2}{*}{$\begin{array}{l}\text { 3. Miller } \\
1998 \\
\\
\begin{array}{c}\text { Target } \\
\text { Pty } \\
\end{array} \\
\end{array}$} & \multirow{2}{*}{\begin{tabular}{|c|} 
4. Miller \\
1998 \\
\\
$\begin{array}{c}\text { Target } \\
\text { Pty }\end{array}$ \\
\end{tabular}} & \multirow{2}{*}{$\begin{array}{c}\text { Brujin } \\
1972 \\
\\
\\
\text { Target } \\
\text { Pty } \\
\end{array}$} & \multirow{2}{*}{\begin{tabular}{|l|}
1. Rein \\
$\&$ Smith \\
1981 \\
\\
$\begin{array}{c}\text { Target } \\
\text { Pty }\end{array}$ \\
\end{tabular}} & \multirow{2}{*}{ 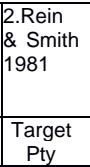 } & \multirow{2}{*}{$\begin{array}{c}\text { Smith } \\
1995 \\
\\
\begin{array}{c}\text { Target } \\
\text { Pty }\end{array} \\
\end{array}$} & \multirow{2}{*}{$\begin{array}{c}\text { Saska } \\
1999 \\
\\
\text { Target } \\
\text { Pty } \\
\end{array}$} & \multirow{2}{*}{\begin{tabular}{|l|} 
1.Gil et \\
al 2001 \\
\\
$\begin{array}{c}\text { Target } \\
\text { Pty }\end{array}$ \\
\end{tabular}} & \multirow{2}{*}{ 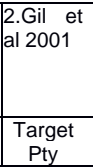 } \\
\hline & & & & & & & & & & & & & & & & & & \\
\hline 1 & 12.78 & 18.00 & 0.71 & 80.10 & 34.80 & 43.45 & 43.35 & 42.39 & \begin{tabular}{|l|l|}
41.17 \\
\end{tabular} & 40.98 & 39.35 & 42.82 & 40.32 & 35.89 & 35.95 & 44.23 & 41.14 & 37.96 \\
\hline 2 & 14.98 & 16.70 & 0.90 & 85.20 & 35.40 & 41.55 & 41.54 & 41.51 & 40.36 & 39.90 & 38.22 & 40.83 & 38.53 & 34.53 & 34.61 & 42.98 & 39.34 & 36.48 \\
\hline 3 & 13.05 & 17.01 & 0.77 & 85.10 & 36.30 & 42.66 & 42.75 & 42.10 & 40.90 & 40.62 & 38.94 & 42.16 & 39.73 & 35.44 & 35.52 & 43.82 & 40.54 & 37.47 \\
\hline 4 & 14.41 & 18.00 & 0.80 & 85.54 & 36.40 & 42.55 & 42.42 & 41.94 & 40.76 & 40.42 & 38.73 & 41.79 & 39.40 & 35.19 & 35.28 & 43.59 & 40.21 & 37.20 \\
\hline 5 & 13.78 & 16.10 & 0.86 & 88.00 & 32.00 & 36.36 & 41.90 & 41.69 & 40.52 & 40.12 & 38.42 & 41.22 & 38.89 & 34.81 & 34.89 & 43.23 & 39.70 & 36.78 \\
\hline 6 & 12.01 & 18.05 & 0.67 & 87.45 & 37.91 & 43.35 & 43.85 & 42.64 & 41.40 & 41.28 & 39.72 & 43.37 & 40.81 & 36.27 & 36.30 & 44.58 & 41.64 & 38.37 \\
\hline 7 & 14.30 & 18.95 & 0.75 & 54.80 & 35.60 & 41.98 & 42.89 & 4217 & 40.97 & 40.70 & 39.04 & 42.31 & 39.86 & 35.55 & 35.62 & 43.91 & 40.68 & 37.58 \\
\hline 8 & 14.12 & 17.05 & 0.83 & 80.10 & 36.45 & 45.51 & 42.16 & 41.81 & 40.64 & 40.27 & 38.57 & 41.51 & 39.14 & 35.00 & 35.08 & 43.41 & 39.95 & 36.99 \\
\hline
\end{tabular}

Table No. 3

Season 2014-15

\begin{tabular}{|c|c|c|c|c|c|c|c|c|c|c|c|c|c|c|c|c|c|c|}
\hline \multirow[t]{2}{*}{$\begin{array}{c}\text { Factory } \\
\# .\end{array}$} & \multirow[t]{2}{*}{$\begin{array}{l}\text { Reducing } \\
\text { Sugar }\end{array}$} & \multirow[t]{2}{*}{$\begin{array}{c}\text { Sulphated } \\
\text { Ash }\end{array}$} & \multirow[t]{2}{*}{$\begin{array}{l}\mathrm{RS} / \mathrm{A} \\
\text { Ratio }\end{array}$} & \multirow[t]{2}{*}{$\begin{array}{l}\text { True } \\
\text { Solids }\end{array}$} & \multirow[t]{2}{*}{ Sucrose } & \multirow[t]{2}{*}{$\begin{array}{l}\text { True } \\
\text { Pty }\end{array}$} & $\begin{array}{c}\text { Foster } \\
1960\end{array}$ & $\begin{array}{c}1 . \\
\text { Miller } \\
1998\end{array}$ & \begin{tabular}{|c|} 
2. Miller \\
1998
\end{tabular} & $\begin{array}{c}\text { 3. Miller } \\
1998\end{array}$ & $\begin{array}{c}\text { 4. Miller } \\
1998\end{array}$ & $\begin{array}{c}\text { Brujin } \\
1972\end{array}$ & $\begin{array}{l}\text { 1.Rein } \\
\& \text { Smith } \\
1981\end{array}$ & $\begin{array}{l}\text { 2.Rein } \\
\& \text { Smith } \\
1981\end{array}$ & $\begin{array}{l}\text { Smith } \\
1995\end{array}$ & $\begin{array}{c}\text { Saska } \\
1999\end{array}$ & $\begin{array}{l}\text { 1.Gil et } \\
\text { al } 2001\end{array}$ & $\begin{array}{l}\text { 2.Gil et } \\
\text { al } 2001\end{array}$ \\
\hline & & & & & & & $\begin{array}{c}\text { Target } \\
\text { Pty }\end{array}$ & $\begin{array}{c}\text { Target } \\
\text { Pty }\end{array}$ & $\begin{array}{l}\text { Target } \\
\text { Pty }\end{array}$ & \begin{tabular}{|c|}
$\begin{array}{c}\text { Target } \\
\text { Pty }\end{array}$ \\
\end{tabular} & \begin{tabular}{|c|}
$\begin{array}{c}\text { Target } \\
\text { Pty }\end{array}$ \\
\end{tabular} & $\begin{array}{c}\text { Target } \\
\text { Pty }\end{array}$ & \begin{tabular}{|c|}
$\begin{array}{c}\text { Target } \\
\text { Pty }\end{array}$ \\
\end{tabular} & $\begin{array}{l}\text { Target } \\
\text { Pty }\end{array}$ & $\begin{array}{c}\text { Target } \\
\text { Pty }\end{array}$ & \begin{tabular}{|c} 
Target \\
Pty \\
\end{tabular} & \begin{tabular}{|c|}
$\begin{array}{c}\text { Target } \\
\text { Pty }\end{array}$ \\
\end{tabular} & $\begin{array}{l}\text { Target } \\
\text { Pty }\end{array}$ \\
\hline 1 & 15.09 & 15.80 & 0.96 & 85.27 & 35.42 & 41.54 & 41.06 & 41.27 & 40.14 & 39.61 & 37.96 & 40.29 & 38.05 & 34.17 & 34.23 & 42.65 & 38.85 & 36.09 \\
\hline 2 & 15.04 & 15.40 & 0.98 & 86.13 & 34.82 & 40.43 & 40.88 & 41.19 & 40.07 & \begin{tabular}{|l|}
39.51 \\
\end{tabular} & 37.88 & 40.10 & 37.88 & 34.04 & 34.10 & \begin{tabular}{|l|}
42.53 \\
\end{tabular} & 38.68 & 35.95 \\
\hline 3 & 14.30 & 19.74 & 0.72 & 84.79 & 35.49 & 41.86 & 43.19 & 42.32 & 41.10 & 40.88 & 39.24 & 42.64 & 40.16 & 35.78 & 35.84 & \begin{tabular}{|l|}
44.12 \\
\end{tabular} & 40.98 & 37.83 \\
\hline 4 & 12.74 & 18.21 & 0.70 & 79.35 & 34.92 & 44.01 & 43.46 & 42.45 & 41.23 & 41.04 & 39.43 & 42.94 & 40.43 & 35.98 & 36.03 & \begin{tabular}{|l|}
44.31 \\
\end{tabular} & 41.25 & 38.05 \\
\hline 5 & 14.90 & 16.70 & 0.9 & 84.80 & 33.50 & 39.50 & 41.58 & 41.53 & 40.38 & 39.93 & 38.24 & 40.87 & 38.57 & 34.56 & 34.64 & \begin{tabular}{|l|}
43.01 \\
\end{tabular} & 39.38 & 36.52 \\
\hline 6 & 12.30 & 16.40 & 0.75 & 86.00 & 38.00 & 44.19 & 42.92 & 42.19 & 40.98 & 40.72 & 39.06 & 42.35 & 39.90 & 35.57 & 35.65 & 43.94 & 40.72 & 37.61 \\
\hline 7 & 13.05 & 17.00 & 0.77 & 85.09 & 36.30 & 42.66 & 42.74 & 42.10 & 40.90 & 40.62 & 38.94 & 42.15 & 39.72 & 35.44 & 35.52 & 43.81 & 40.54 & 37. \\
\hline
\end{tabular}


Table No. 3

\begin{tabular}{|c|c|c|c|c|}
\hline \multirow{2}{*}{$\begin{array}{l}\text { Factory } \\
\text { No. }\end{array}$} & \multirow[t]{2}{*}{ RS/A Ratio } & \multirow[t]{2}{*}{ True Purity } & \multirow{2}{*}{$\begin{array}{c}\text { New Equation } \\
\text { Target Pty }\end{array}$} & \multirow{2}{*}{$\begin{array}{c}\text { Final Molasses TPD } \\
\text { PPD = Target Purity-Target Purity }\end{array}$} \\
\hline & & & & \\
\hline 1 & 0.99 & 36.79 & 40.95 & -4.16 \\
\hline 2 & 0.92 & 35.98 & 41.41 & -5.43 \\
\hline 3 & 0.65 & 44.36 & 43.57 & 0.79 \\
\hline 4 & 0.79 & 42.87 & 42.38 & 0.49 \\
\hline 5 & 0.82 & 40.97 & 42.12 & -1.14 \\
\hline 6 & 0.65 & 43.23 & 43.57 & -0.34 \\
\hline 7 & 0.84 & 45.45 & 42.01 & 3.44 \\
\hline 8 & 0.76 & 42.73 & 42.62 & 0.11 \\
\hline 9 & 0.69 & 43.18 & 43.19 & -0.02 \\
\hline 10 & 0.75 & 43.93 & 42.72 & 1.21 \\
\hline 11 & 0.73 & 43.37 & 42.87 & 0.50 \\
\hline 12 & 0.66 & 43.11 & 43.48 & -0.37 \\
\hline 13 & 0.97 & 43.65 & 41.06 & 2.58 \\
\hline 14 & 0.88 & 39.52 & 41.65 & -2.13 \\
\hline 15 & 1.03 & 37.20 & 40.65 & -3.46 \\
\hline 16 & 0.96 & 41.54 & 41.16 & 0.38 \\
\hline 17 & 0.98 & 40.43 & 41.02 & -0.59 \\
\hline 18 & 0.72 & 41.86 & 42.91 & -1.05 \\
\hline 19 & 0.70 & 44.01 & 43.13 & 0.88 \\
\hline 20 & 0.71 & 43.45 & 43.04 & 0.41 \\
\hline 21 & 0.90 & 41.55 & 41.56 & -0.01 \\
\hline 22 & 0.77 & 42.66 & 42.55 & 0.11 \\
\hline 23 & 0.80 & 42.55 & 42.28 & 0.28 \\
\hline 24 & 0.86 & 36.36 & 41.85 & -5.49 \\
\hline 25 & 0.67 & 43.35 & 43.45 & -0.10 \\
\hline 26 & 0.75 & 41.98 & 42.66 & -0.68 \\
\hline 27 & 0.83 & 45.51 & 42.06 & 3.44 \\
\hline 28 & 0.89 & 39.50 & 41.59 & -2.09 \\
\hline 29 & 0.75 & 44.19 & 42.69 & 1.49 \\
\hline 30 & 0.77 & 42.66 & 42.54 & 0.12 \\
\hline
\end{tabular}

\section{DISCUSSION}

Now applying this new formula at all thirty molasses exhaustion tests following results are obtained

\section{REFERENCES}

Baikow, V.E., 1967. Manufacturing and refining of raw cane sugar. Elsevier publishing company, New York.

Hubert Olbrich. 1963. The Molasses, Berlin, Germany.

Hugot, E., 1986. Handbook of sugarcane engineering. Saint-Denis (Re-Union).

Khan, H. R., 2000. Fabrication Practices of Boiling House of a sugar Factory. Karachi, Pakistan. 62-63.

Rein, P.W., 2007. Cane Sugar Engineering. Verlag Dr. Albert Bartens KG, Berlin, Germany page 455-465.

Tahir, A.A., 2013. Assessment of Hidden Sugar Losses in Cane Sugar Factory, 47th Annual Convention of PSST, Rawalpindi. 